\title{
CONTROLE DA QUALIDADE FÍSICA DO SINTER PARA ALTO FORNO*
}

\section{Resumo}

\author{
Cristiane Vilma Rocha Galiazzi ${ }^{1}$ \\ Erick Torres Bispo dos Santos ${ }^{2}$ \\ Wagner Luis Garcia Silveira ${ }^{3}$ \\ Luiz Henrique Curado Ribeiro ${ }^{4}$ \\ Ronaldo Oliveira Silva ${ }^{5}$ \\ Bruno Pinheiro da Silva ${ }^{6}$
}

As matérias primas para Alto Forno devem ter qualidade física adequada para melhor controle do processo e estabilidade operacional. A distribuição granulométrica e consequentemente sua estabilidade são importantes, pois interferem na porosidade e permeabilidade no interior do Alto-Forno, criando assim uma condição adequada e específica de marcha com reflexo na estabilidade da produção de gusa. A Sinterização da ThyssenKrupp Companhia Siderúrgica do Atlântico (TKCSA) foi projetada sem britagem secundária, devido à fase de ajustes no processo operacional e baixa produtividade havia dificuldade para controlar a fração grossa do sinter (>50 mm), impactando na distribuição granulométrica do sinter. Visando reduzir os desvios da qualidade física, estudou-se a melhor relação da altura de camada $x$ velocidade da esteira encontrando um ponto de equilíbrio entre os níveis de produção e qualidade especificada que, juntamente com o início da operação do britador secundário, resultou na garantia da qualidade física do sinter para os Altos Fornos. Este trabalho tem como objetivo apresentar 0 desenvolvimento e resultados deste estudo.

Palavras-chaves: Sinterização; Distribuição granulométrica do sinter; Altura de camada da máquina; Britagem secundária.

\begin{abstract}
The raw materials of Blast Furnace must have physical quality adequate to improve process control and operational stability. The size distribution and consequently the stability are important because affect burden porosity and blast furnace permeability, creating adequate condition to have operational stability. Sinter Plant at ThyssenKrupp Companhia Siderúrgica do Atlântico (TKCSA), was projected without secondary crusher and due to phases of adjust process and low productivity there was difficult to control the sinter size (>50mm), affecting in granulometry distribution. In order to reduce deviation of physical quality, was studied improve bedding height ratio versus sinter machine speed finding a better balance between production levels and agreed quality, in the same time started operation of secondary crusher, resulting in assurance sinter physical quality to blast furnaces. The goal of this work is show the development and results of this study.
\end{abstract}

PHYSICAL QUALITY CONTROL OF SINTER FOR BLAST FURNACE

Keywords: Sintering; Size distribution; Bed height; Secondary crusher.

1 Engenheiro de Materiais, M.Sc, Engenheiro de Processo, Unidade Técnica da Redução, TKCSA, Rio de Janeiro, Brasil.

2 Engenheiro Metalurgista, M.Sc, Gerente de Unidade Técnica, Unidade Técnica da Redução, TKCSA, Rio de Janeiro, Brasil.

3 Engenheiro Metalurgista, Engenheiro de Processo, Unidade Técnica da Redução, TKCSA, Rio de Janeiro, Brasil.

4 Engenheiro Metalurgista, Engenheiro Metalúrgico, Unidade Técnica da Redução, TKCSA, Rio de Janeiro, Brasil.

5 Técnico Metalurgista, Técnico Especialista, Sinterização Operação, TKCSA, Rio de Janeiro, Brasil.

6 Graduando em Engenharia química, Especialista de Alto Forno, Unidade Técnica da Redução, TKCSA, Rio de Janeiro, Brasil.

* Contribuição técnica ao 44은 Seminário de Redução de Minério de Ferro e Matérias-primas, $15^{\circ}$ Simpósio Brasileiro de Minério de Ferro e $2^{\circ}$ Simpósio Brasileiro de Aglomeração de Minério de Ferro, 15 a 18 de setembro de 2014, Belo Horizonte, MG, Brasil. 


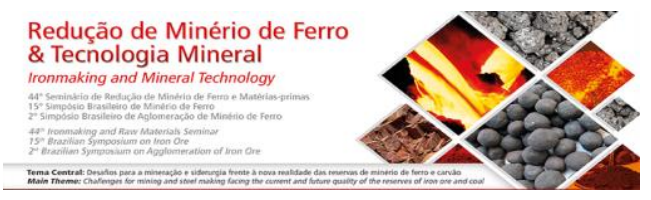

\section{INTRODUÇÃO}

A estabilidade operacional do Alto Forno depende fortemente da qualidade física e química das matérias primas a serem enfornadas, o sinter nos Altos Fornos da TKCSA contribui com $55-65 \%$ da carga metálica. O controle do processo de sinterização é fundamental para alcançar a qualidade elevada e estável do sinter, bem como a produtividade [1,2]. Muitos estudos têm sido focados na correlação teórica entre altura da camada, produtividade e qualidade do sinter [3].

O processo de sinterização deve ser cuidadosamente ajustado, de modo que a zona de combustão alcance a parte inferior da camada antes da descarga da máquina, conhecido como o controle do BTP (Burn Through Point). Muitos parâmetros precisam ser ajustados, incluindo a velocidade e a quantidade da mistura a ser carregada. No entanto, a fim de obter bons resultados, o controle de sinterização requer ajuste fino dos parâmetros de processo [4]. Além disso, a estrutura do sinter e suas características também dependem da matéria-prima (química, física, distribuição granulométrica, etc.) [5].

A TKCSA iniciou a operação das plantas em maio de 2010 e está em processo rump up dos níveis de produção. A sinterização possui uma máquina de sínter com 456 $\mathrm{m}^{2}$ e hoje opera em média com $87 \%$ da capacidade de projeto $(14.450 \mathrm{t} / \mathrm{d})$. Este trabalho foi fundamental para controle da qualidade física do sinter sem a britagem secundária, principalmente para operar em baixa produtividade e na fase de estabilização operacional dos processos de uma nova usina Siderúrgica.

\section{MATERIAIS E MÉTODOS}

\subsection{Definição de Parâmetros para Controle do Processo}

Os parâmetros operacionais (velocidade da esteira $X$ altura da camada) foram definidos por meio de correlações com os resultados da qualidade física do sinter (fração > $50 \mathrm{~mm}$ ), obtendo-se o maior controle da velocidade (ou facilidade) com que a camada de sinterização será atravessada pelo fluxo de ar/gás, ou seja, o controle da velocidade de sinterização (Equação 1) para cada ritmo de produção. Para obter a velocidade de sinterização calculada (Figura 1), foi utilizado o comprimento total da área de sucção da máquina de sínter 114 m, o BTP foi fixado na posição de 109 m.

Onde:

$$
V S=V M S *(\text { Htotal }- \text { Hbedding }) / C
$$

VS = Velocidade de Sinterização ( $\mathrm{mm} / \mathrm{min})$

VMS $=$ Velocidade da Máquina de Sinter $(\mathrm{m} / \mathrm{min})$

$\mathrm{H}_{\text {total }}=$ Altura total do leito $(\mathrm{mm})$

$\mathrm{H}$ bedding = Altura da camada de forração $(\mathrm{mm})$

$\mathrm{C}=$ Comprimento da área de sucção $(\mathrm{m})$ 
Redução de Minério de Ferro
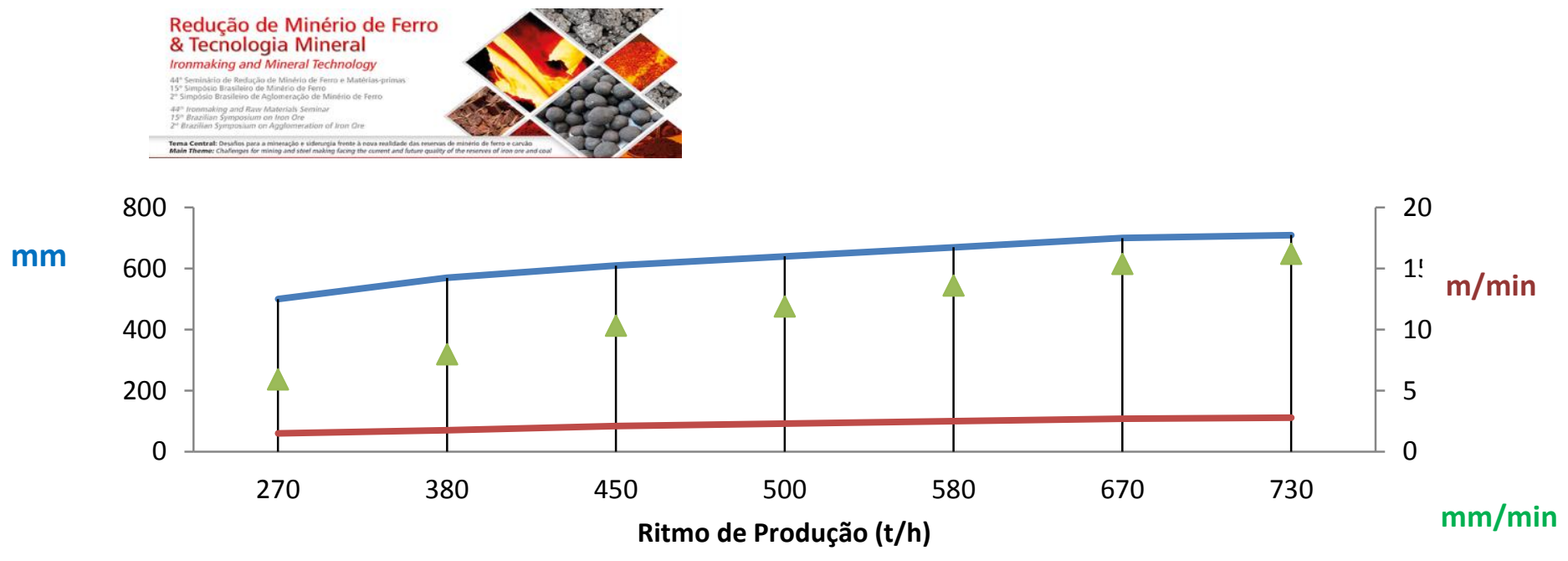

Altura da camada - $\mathrm{mm} \longrightarrow$ Velocidade da Máquina $(\mathrm{m} / \mathrm{min}) \quad \Delta$ Velocidade de Sinterização $(\mathrm{mm} / \mathrm{min})$

Figura 1. Altura da camada $x$ velocidade da máquina para cada ritmo operacional.

\subsection{Britador Secundário}

O sistema de britagem secundário é importante para garantia e controle da distribuição granulométrica do sinter principalmente para atuar na baixa produtividade e/ou instabilidades operacionais que afetam a qualidade do produto.

$\mathrm{O}$ start up do sistema de britagem secundária (Figura 2) iniciou-se em set/13. $\mathrm{O}$ sistema recebe o sinter produto com a fração $>20 \mathrm{~mm}$ e realiza o pré-peneiramento em $63 \mathrm{~mm}$ onde toda a fração $>63 \mathrm{~mm}$ do sinter é britada, garantindo então o controle da fração $>50 \mathrm{~mm}$ de acordo com a especificação para os Altos Fornos (5\% máximo).

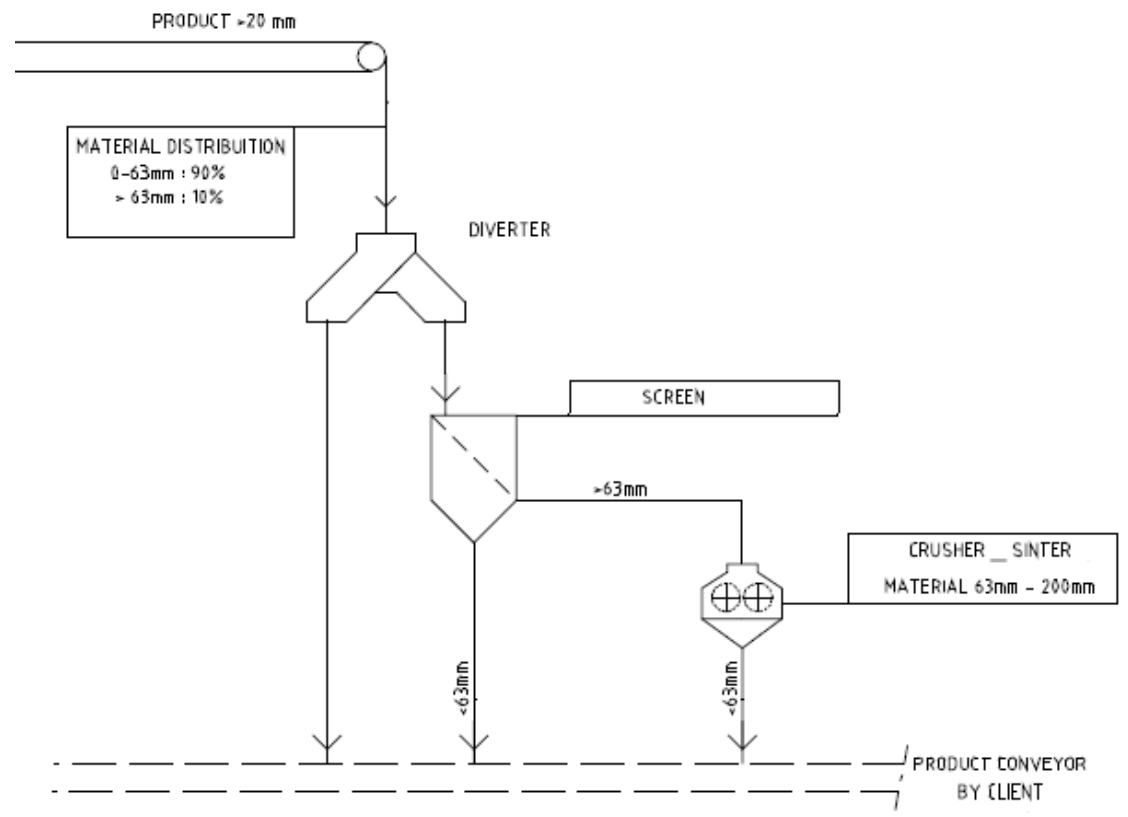

Figura 2. Sistema de britagem secundária da Sinterização TKCSA.

\subsection{Controle da Permeabilidade no Alto Forno}

O controle da distribuição granulométrica da carga metálica do Alto Forno é fundamental para controle do processo, pois impacta diretamente na porosidade da carga e por consequência na permeabilidade (Figura 3). Assim a redução na

* Contribuição técnica ao $44^{\circ}$ Seminário de Redução de Minério de Ferro e Matérias-primas, $15^{\circ}$ Simpósio Brasileiro de Minério de Ferro e $2^{\circ}$ Simpósio Brasileiro de Aglomeração de Minério de Ferro, 15 a 18 de setembro de 2014, Belo Horizonte, MG, Brasil. 
dispersão granulométrica traz homogeneidade de carga, facilitando o controle e evitando a impermeabilização de zonas no forno.

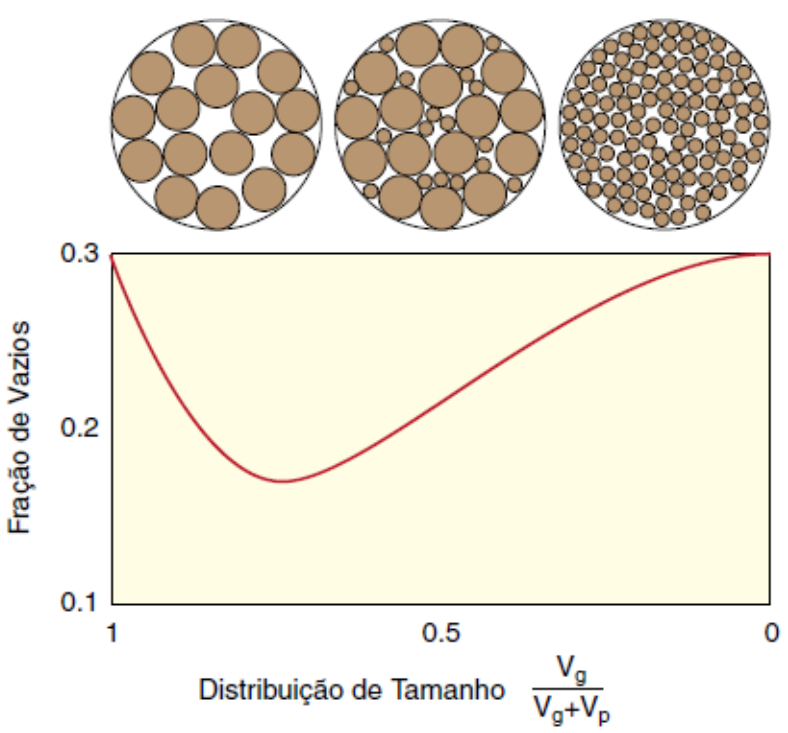

Figura 3. A permeabilidade ao fluxo de gás depende da fração de vazios, que por sua vez depende da taxa entre partículas menores e maiores [6].

\subsection{Uniformidade das cargas nos Altos Fornos}

O sistema de carregamento adotado nos fornos da TKCSA foi o bell-less (Figura 4) com calha distribuidora e controladora de fluxo (LMG), os fornos com calha distribuem a massa carregada através de anéis e como normalmente a velocidade angular da calha é constante a simetria do carregamento depende do ajuste adequado da vazão mássica da carga distribuída. Assim uma elevada dispersão granulométrica interfere no escoamento granular criando perturbações no sistema de carregamento e por consequência prejudicando o controle do fluxo gasoso.

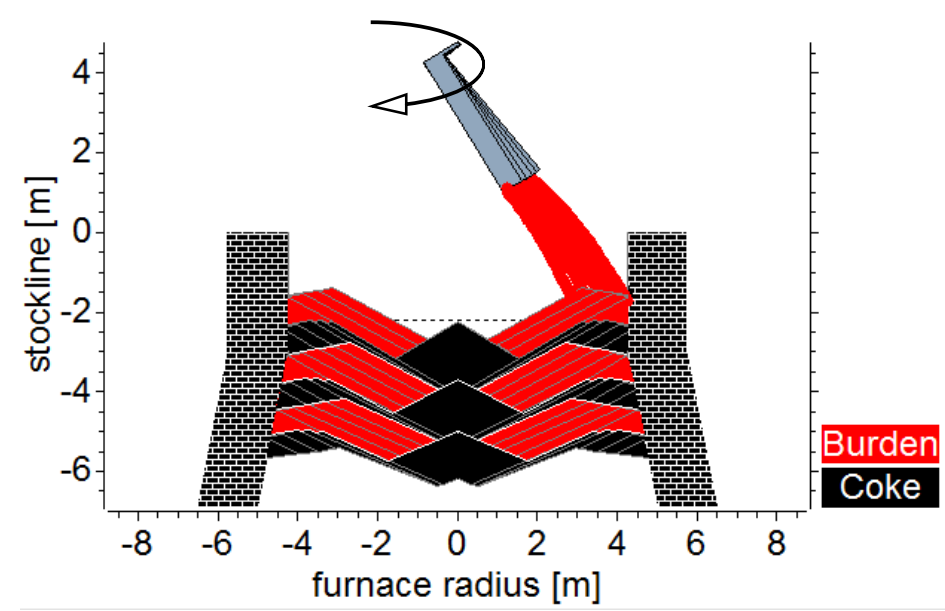

Figura 4. Sistema de carregamento com calha distribuidora.

* Contribuição técnica ao 44 Seminário de Redução de Minério de Ferro e Matérias-primas, 15은 Simpósio Brasileiro de Minério de Ferro e 2o Simpósio Brasileiro de Aglomeração de Minério de Ferro, 15 a 18 de setembro de 2014, Belo Horizonte, MG, Brasil. 


\section{RESULTADOS E DISCUSSÃO}

\subsection{Distribuição Granulométrica do Sinter}

\subsubsection{Caracterização da partícula}

Foram avaliados três modelos de distribuição granulométrica para a caracterização do Sinter, sendo esses o GGS (Gates-Gaudin-Shaumann), Sigmóide e RRB (RosinRammler-Bennet). Equações 2, 3 e 4 respectivamente.

$$
\begin{array}{r}
y=\left(\frac{d}{K}\right)^{m} \\
y=\frac{1}{1+\left(\frac{k}{d}\right)^{m}} \\
y=1-\exp \left[-\left(\frac{d}{k}\right)^{m}\right]
\end{array}
$$

Sendo o Sigmoide que melhor descreveu os dados discretos da granulometria do sínter.

Onde:

$$
y=\frac{1}{1+\left(\frac{k}{d}\right)^{m}}
$$

(Sigmóide)

y = Fração granulométrica acumulada menor que o diâmetro de referência.

$\mathrm{d}=$ Diâmetro de referência.

$\mathrm{K}$ e $\mathrm{m}$ = Parâmetros de ajuste do modelo.

Evolução nas curvas de distribuição do sinter.

Conforme apresentado na Figura 5 as distribuições discretas do sinter foram modeladas para três diferentes períodos (Tabela 1):

Tabela 1. Apresentação dos itens contidos em cada período.

\begin{tabular}{lccc}
\hline & Período 1 & Período 2 & Período 3 \\
\hline Padronização dos Parâmetros Operacionais & Não & Sim & Sim \\
\hline Britagem Secundária & Não & Não & Sim \\
\hline
\end{tabular}




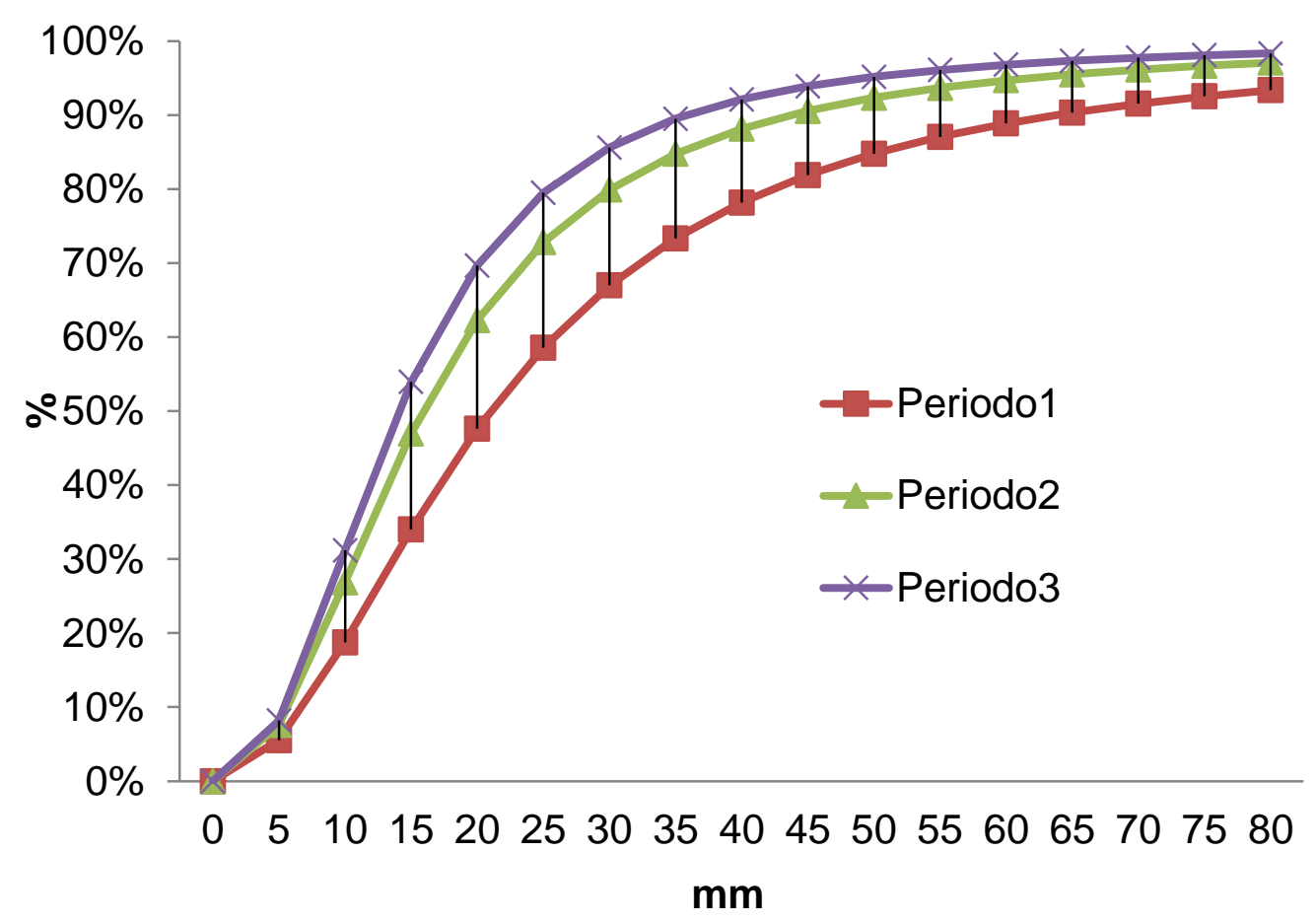

Figura 5. Distribuição acumulada do sinter (Sigmóide) para cada período.

\subsubsection{Controle da fração $>50 \mathrm{~mm}$ do sinter.}

A Figura 6 mostra os resultados diários da fração $>50 \mathrm{~mm}$ nos três períodos. Como pode ser visto, comparando os períodos 1 e 2 , é mostrado claramente a redução da fração $>50 \mathrm{~mm}$ do sinter abaixo do limite superior de controle (5\%) e a redução no desvio padrão. Com o britador secundário em operação, no período 3, melhores resultados foram obtidos.

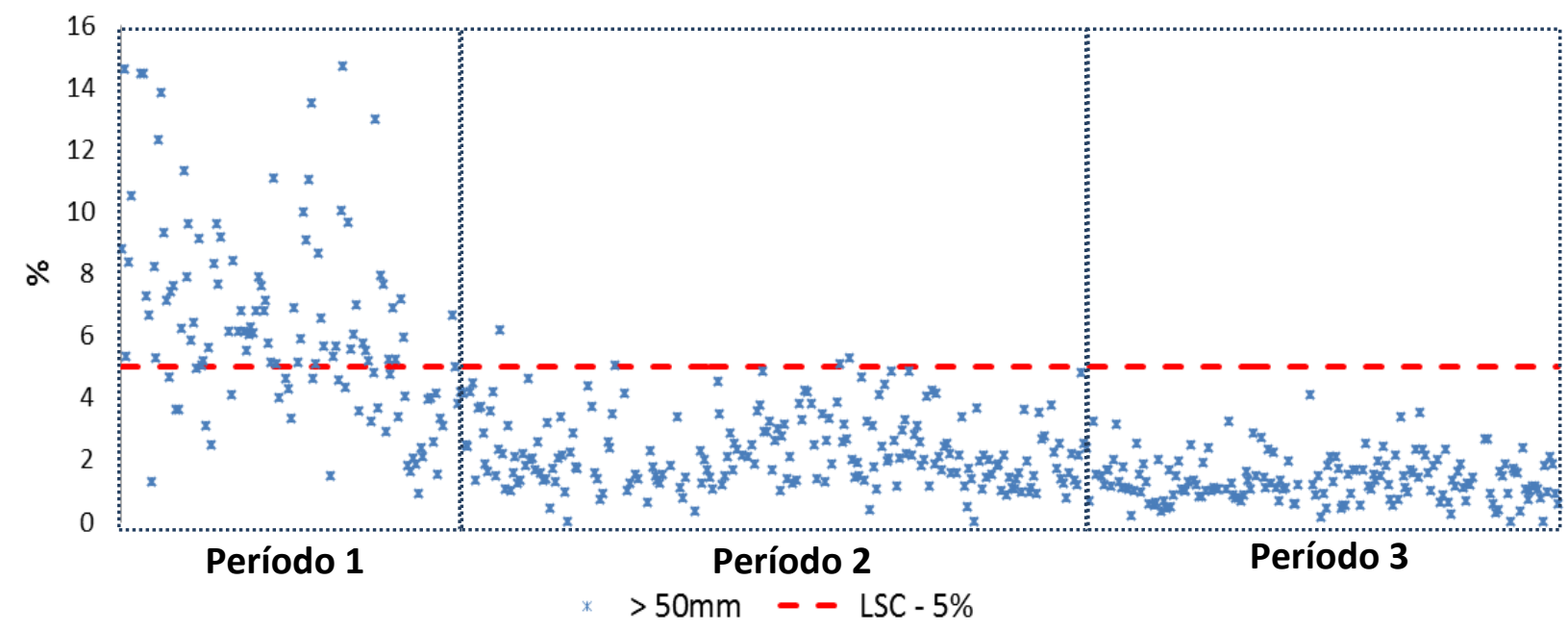

Figura 6. Resultados diários da fração > $50 \mathrm{~mm}(\%)$ do sínter em cada período.

A Figura 7 mostra a evolução dos resultados obtidos em cada período.

* Contribuição técnica ao 44은 Seminário de Redução de Minério de Ferro e Matérias-primas, 15ํ Simpósio Brasileiro de Minério de Ferro e 2o Simpósio Brasileiro de Aglomeração de Minério de Ferro, 15 a 18 de setembro de 2014, Belo Horizonte, MG, Brasil. 


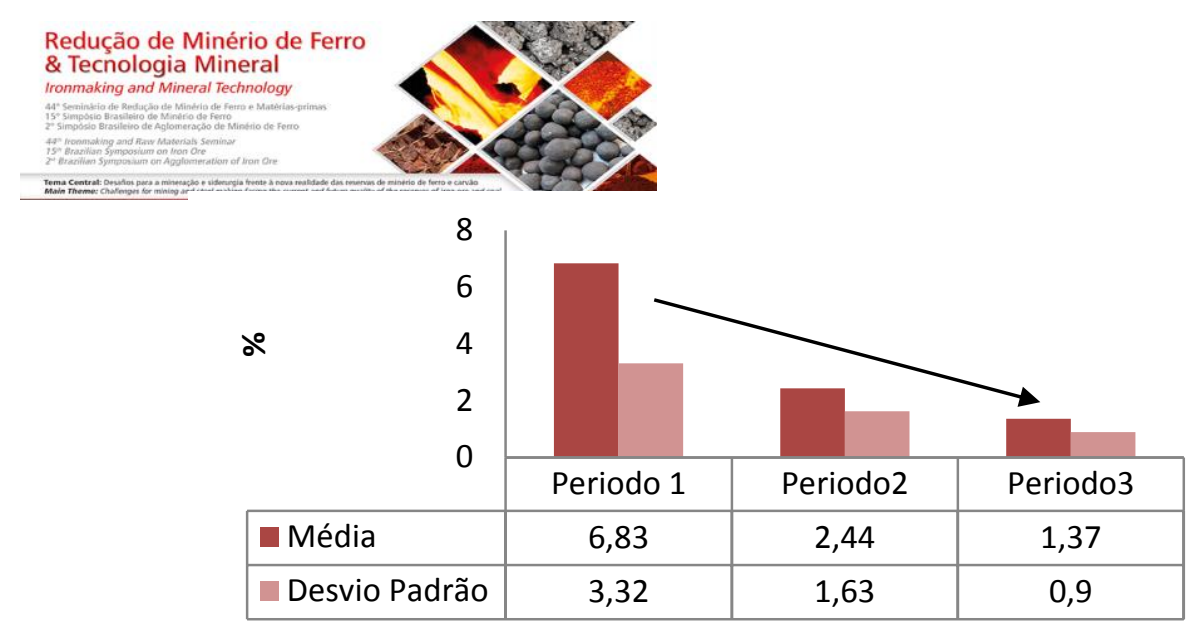

Figura 7. Percentual da fração > $50 \mathrm{~mm}$ e desvio padrão em cada período.

\section{CONCLUSÃo}

Em virtude dos dados e fatos apresentados, pode-se concluir que o controle da fração > $50 \mathrm{~mm}$ do sinter foi obtido com o melhor controle do processo de sinterização para cada ritmo de produção, por meio da relação altura da camada $x$ velocidade da máquina. Com a instalação do britador secundário, o ajuste fino foi obtido com a garantia da fração $>50 \mathrm{~mm}$ abaixo de $5 \%$. Foi observada nos resultados a elevação na fração $<5 \mathrm{~mm}$, porém, abaixo do limite superior de controle (5\%). Não foi observado perda de rendimento do sínter nem aumento no consumo de combustível nos períodos 2 e 3.

Com a nova configuração do sínter, foi possível obter melhores ajustes nos sistema de peneiramento, distribuição de carga e um controle mais adequado na distribuição dos gases no forno, possibilitando a otimização do processo que contribuiu expressivamente na redução de combustíveis.

\section{REFERÊNCIAS}

1 Ram Pravesh BHAGAT, Uday Shankar CHATTORAJ and Samir Kumar SIL (Late). Porosity of Sinter and Its Relation with the Sintering Indices. ISIJ Internacional, 2006; 46 (11): 1728-1730.

2 Andreas Franzen, Süleyman Bastürk, Joachim Janz, Jörn Pufpaff. Model-based control of the position of the burn-through point of the iron ore sinter process. METEC InSteelCon, Düsseldorf, 27 June - 1 July 2011

3 Myung-Suk Choi, Gi-Ryong Yoo, Seok-Ki Kim, Han-Ga-Ram Yang, High Bed Height Operation with Small-sized Sintering Machine. METEC InSteelCon, Düsseldorf, 27 June 1 July 2011

4 Rubén Usamentiaga, Julio Molleda, Daniel F. Garcia, Francisco G. Bulnes. Monitoring Sintering Burn-Thorough Point Using Infrared Thermography. Sensors 2013, 13, 1028710305. August 2013.

5 Tekkalakote UMADEVI, Angalakuditi Brahmacharyulu, Ajay Kumar Roy, Pradipta Chandra Mahapatra, Manjunath Prabhu and Madhu Ranjan. Influence of Iron Ore Fines Feed Size on Microstructure,Productivity and Quality of Iron Ore Sinter. ISIJ International, 2011; 51(6): 922-929

6 Maarten Geerdes, Hisko Toxopeus, Cor van der Vliet. Modern Blast Furnace IronMaking an introduction. $2^{\circ}$ Edição. 2009. 